\title{
Wavelet Robust Control by Fuzzy Boundary Layer via Time-variant Sliding Surface
}

\author{
Majid Yarahmadi ${ }^{1}$, Seyed Mehdi Karbassi ${ }^{1}$, Ahmad Mirzaei ${ }^{2}$ \\ ${ }^{1}$ Faculty of Mathematics, Yazd University, Yazd, 89195-741, Iran. \\ myarahmadi@yazduni.ac.ir, smkarbassi@yazduni.ac.ir \\ ${ }^{2}$ Faculty of Electrical and Computer Engineering, Yazd University, Yazd, 89195-741, Iran. \\ mirzaei@yazduni.ac.ir
}

\begin{abstract}
In this paper, a new wavelet robust control by fuzzy boundary layer via time-variant sliding surface (WRCFBL) for an uncertain nonlinear system is presented. New terminologies, rejection parameter and rejection regulator, for designing a time-variant sliding surface are defined. The time variant sliding surface operates as an adaptive filter. Wavelet network is used to design an indirect controller. An adjustable control gain parameter, rejection parameter and the wavelet network coefficients are on-line tuned. Instead of saturation function a hyperbolic tangent function is used. Also, a fuzzy system that adopts absolute value of sliding surface as input and the boundary layer parameter as output is defined. This fuzzy system tunes the boundary layer width. Control system stability is guaranteed by using the Lyapunov method. The proposed method attenuates efficiently the effects of the system uncertainties and un-modelled frequencies. Also, the chattering phenomenon is completely eliminated. In addition, three theorems and one lemma, which facilitate design of the proposed controller, are proved. Also, a simulation example is presented to illustrate the performances and the advantages of the proposed method.
\end{abstract}

Keywords: robust control, wavelet networks, time-variant sliding surface, rejection regulator, fuzzy boundary layer

\section{Introduction}

There are several techniques to control of an uncertain nonlinear system. A typical approach is sliding mode control technique [14]. In the sliding mode technique, the proper transformation of tracking errors to generalize errors is introduced, so that $n$ order tracking problem can be transformed into an equivalent first order stabilization problem [16]. The sliding-mode control employs a discontinuous control to derive the system state to reach and maintain its motion on sliding surface. The discontinuity in the control action provides the chattering and the un-modeled frequencies may be activated, which are undesirable in application. To avoid these drawbacks, the boundary layer technique is exploited [14]. For achieving the better tracking performance a varying boundary layer is considered. In [9], the selftuning laws based on the bounded modeling error, for adjusting the boundary layer width and the other parameters have also been proposed. Furthermore, for calculating the control gain parameter, the difference functions $\Delta f$ and $\Delta g$ must be obtained, that is a drawback. The auto-tuning neurons computation for designing the sliding-mode control [3] and the fuzzy adjusting method for finding the suitable boundary layer width [12] are used. Most practical systems are non-linear and complex in nature with uncertain dynamics that may not be easily modeled mathematically. For this purpose, the identification methods are usually exploited [1], [2], [11], [17]. A direct adaptive fuzzy sliding mode control for uncertain nonlinear systems was presented in [13]. The GA-based fuzzy sliding mode controller with modified adaptive laws for robust control of an uncertain nonlinear plant has also been presented [4]. Recently, wavelets have led to advanced tools in many scientific and application research areas [5]. Multiscale analysis, synthesis properties and the learning abilities of neural wavelet networks, for approximation of nonlinear functions are well established [6], [15], [18]. In the literature only time-invariant sliding surface has been studied extensively. Here for the first time, a new case of time-variant sliding equation is presented. For this purpose, the rejection regulator based on a parameter that is called "rejection parameter" is defined. For objectively choosing the coefficients of error states in sliding equation rejection regulator is used. By tuning the rejection parameter, we can adjust the break frequency bandwidth and also the coefficients of error states in sliding equation. Such sliding equation, as a chain of $(n-1)$ adaptive first-order low-pass filters, rejects all un-modeled frequencies. The tracking precision is not guaranteed by using the saturation function. Therefore, instead of 
saturation function, a hyperbolic tangent function is used. Also, a fuzzy system, that adopts absolute value of sliding surface as input and the boundary layer parameter as output, is defined. This fuzzy system tunes the boundary layer width. Control system stability is guaranteed by using the Lyapunov method. For facilitating the design of the proposed controller, three theorems and one lemma are proved. This paper is organized as follows. Problem formulation is presented in section 2 . In section 3 , sliding mode control with new terminologies as rejection parameter and rejection regulator is presented. Two theorems and one lemma complete this section. Section 4 has two subsections. In the first subsection a wavelet network system is described briefly and a theorem for presenting the tuning laws is explained. This theorem adjusts the wavelet network coefficients, rejection parameter and control gain parameter. In the last subsection of the section 4, structure of a fuzzy system, to design a fuzzy boundary layer, is presented. An example to illustrate the effectiveness of the proposed method is presented in section 5. Finally, the paper is concluded in section 6 .

\section{Problem Formulation}

Consider the $n$-th order nonlinear dynamical system defined by the following state equation

$x^{(n)}=f\left(x, \dot{x}, \ldots, x^{(n-1)}\right)+g\left(x, \dot{x}, \ldots, x^{(n-1)}\right) u+d$

where $f$ and $g$ are unknown bounded nonlinear functions, and $u \in R$ is control input of the system and $d$ is the external bounded disturbance. Let

$\mathbf{x}=\left[x_{1}, x_{2}, . ., x_{n-1}, x_{n}\right]=\left[x, \dot{x}, \ldots, x^{(n-2)}, x^{(n-1)}\right] \in R^{n}$ and $\quad \mathbf{x}_{d}=\left[x_{d}, \dot{x}_{d}, \ldots x_{d}^{(n-1)}\right] \in R^{n}$, where $\mathbf{x}$ and $\mathbf{x}_{d}$ are state vector and desired trajectory of the system, respectively. Assume that $\mathbf{x}$ is available. The control objective is to find a controller so that the state $\mathbf{x}$ can track the desired trajectory $\mathbf{x}_{d}$. Thus we define $\mathrm{e}=\mathrm{x}-\mathrm{x}_{d}=\left[e, \dot{e}, \ldots, e^{(n-1)}\right]$, such that $e=x-x_{d} \in R$. For achieving the control objective, the tracking error $e=x-x_{d}$ must be attenuated to an arbitrary small residual tracking error independent of the uncertainties of the system. The slidingmode control is a method based on defining a sliding surface in the error space passing. Consider a sliding function as, $s=\mathbf{k}^{T}$ e. In particular, $\quad \mathbf{k}=\left[k_{0}, k_{1}, \ldots, k_{n-1}\right]^{T} \in R^{n} \quad$ is chosen such that all roots of the characteristic polynomial:

$$
P(\omega)=k_{0} \omega^{(n-1)}+k_{1} \omega^{(n-2)}+\ldots+k_{n-1}
$$

corresponding to the sliding equation:

$k_{0} e^{(n-1)}+k_{1} e^{(n-2)}+\ldots+k_{n-1} e=0$

lie in the open left-half plane. By choosing the Lyapunov function candidate

$V=\frac{1}{2} s^{2}$

With $V(0)=0 \quad$ a controller $u$ is given such that each state Lyapunov condition holds for system stability (sliding condition) [14]:

$\dot{V}=\frac{1}{2} \frac{d}{d t} s^{2} \leq-\eta|s|, \quad \eta>0$

The other equivalent relation of the reaching condition is:

$s \dot{s} \leq-\eta|s|$, or $\dot{s} \operatorname{sgn}(s) \leq-\eta, \quad \eta>0$.

An alternative definition of the sliding function is [14]:

$s=\left(\frac{d}{d t}+\lambda\right)^{n-1} e$

Two steps for designing the sliding-mode control exist. The first step is the selection of parameter $\lambda$ such that all un-modeled frequencies of the system are rejected. The other step is to find a control law such that the reaching condition (6) is satisfied.

\section{Sliding-mode Control and Rejection Regulator}

By using the Laplace transformation, the linear differential equation (7) can be considered as a chain of $(n-1)$ first-order low-pass filters with break frequency bandwidth. Such that the scalars $s$ and $e$ are input and output of the filters, respectively. Figure 1 shows pictorially this filter. 


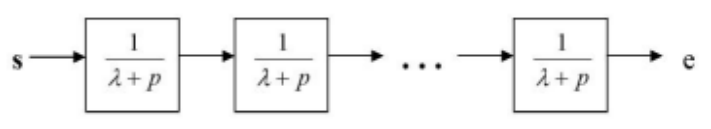

Figure 1. Sliding equation as chain of low-pass filters.

So that $p$ is Laplace operator. The parameter $\lambda$ should be selected such that all unmodeled frequencies of the system are rejected. Let $G(p)=1 /(\lambda+p)$ be a first order low-pass filter corresponding to $\mathrm{Eq}$ (7). In order to reject all un-modeled frequencies, the inequalities $\lambda<p$ or $\lambda<v$ must be satisfied, where $v$ is defined as

$\lambda<v=\inf \left\{\sigma_{u f}: \sigma_{u f}\right.$ are unmodeled frequencies $\}$

In the following, a theorem for objectively choosing the coefficients of error states in sliding equation is presented.

Theorem 1: If $\omega_{i}$ are roots of the polynomial (2), $v$ is the same notation as defined in (8). $\omega^{*}=\max \left\{\left|\omega_{i}\right|: P\left(\omega_{i}\right)=0, \omega_{i}<0, i=1,2, . . n-1\right\}$ and $\delta$ is an arbitarary real positive number (rejection parameter) such that $\delta<v$ then by choosing $\omega^{*}=v-\delta$ and

$k_{j}=\left(\begin{array}{c}n-1 \\ j\end{array}\right)\left(\omega^{*}\right)^{j}, \quad$ for $j=0, \ldots, n-1$,

we have $\lambda<v$, i.e., the first-order low filters with break frequency bandwidth $\lambda$, as defined in Eq. (7) reject all un-modeled frequencies of the system.

Proof. Let $s=\mathbf{k}^{T} \mathbf{e}=0$ be sliding equation in the error space passing, such that $k_{0}=1$. Else the sliding equation is divided by $k_{0}$. The coefficients $k_{j}$, for $j=1,2, \ldots, n-1$, must be chosen such that all roots of the characteristic polynomial of the sliding equation (7):

$P(\omega)=\omega^{(n-1)}+k_{1} \omega^{(n-2)}+\ldots+k_{n-1}$

lie in the open left-half plane. By using the well known relationship between coefficients and roots of the polynomials [7], we have

$k_{1}=-\sum_{i=1}^{n-1} \omega_{i}$ $k_{2}=\sum_{i, l=1, i \neq l}^{n-1} \omega_{i} \omega_{l}$

$k_{n-1}=(-1)^{(n-1)} \Pi_{i=1}^{(n-1)} \omega_{i}$

since

$\omega^{*}=\max \left\{\left|\omega_{i}\right|: P\left(\omega_{i}\right)=0, \omega_{i}<0, i=1,2, . . n-1\right\}$, therefore

$k_{1} \leq\left(\begin{array}{c}n-1 \\ 1\end{array}\right) \omega^{*}$

$k_{2} \leq\left(\begin{array}{c}n-1 \\ 2\end{array}\right)\left(\omega^{*}\right)^{2}$

and finally

$k_{n-1} \leq\left(\omega^{*}\right)^{n-1}$

On the other hand, the sliding surface can be considered as a chain of $(n-1)$ first-order low-pass filters. Therefore,

$s=\left(\frac{d}{d t}+\lambda\right)^{n-1} e=\mathbf{k}^{\mathbf{T}} \mathbf{e}$

or equivalently:

$s=\left(\frac{d}{d t}+\lambda\right)^{n-1} e=\sum_{j=0}^{n-1}\left(\begin{array}{c}n-1 \\ j\end{array}\right)(\lambda)^{j} e^{(n-j-1)}=$

$e^{(n-1)}+k_{1} e^{(n-2)}+\ldots+k_{n-1} e$

where

$k_{j}=\left(\begin{array}{c}n-1 \\ j\end{array}\right)(\lambda)^{j} \quad$ for $j=1, \ldots, n-1$.

Equations (13-15) and (18) yield:

$\left(\begin{array}{c}n-1 \\ j\end{array}\right)(\lambda)^{j} \leq\left(\begin{array}{c}n-1 \\ j\end{array}\right)\left(\omega^{*}\right)^{j}$

Therefore, we can write

$\lambda \leq \omega^{*}$.

Since $\omega^{*}=v-\delta$ such that $\delta$ is a positive real number $(\delta<v)$, we have

$\lambda<v$

and this completes the proof.

Definition: Define the rejection regulator in the following form: 


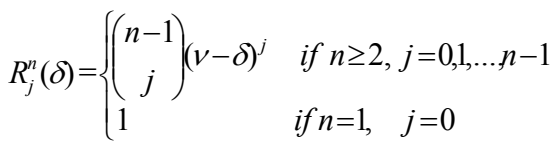

where $\delta$ and $v$ are rejection parameter and the same notations as defined in (8), respectivelly.

Now, according to the Eq. (22), consider the sliding function in the following form:

$s=\sum_{j=0}^{n-1} R_{j}^{n}(\delta) e^{(n-j-1)}$

In most practical systems the nonlinear functions $f$ and $g$ are unknown. Now let $\hat{f}$ and $\hat{g}$ be estimates of functions $f$ and $g$, respectively. In order to design a controller, that establishe the sliding condition, we need the following assumption.

Assumption I: The upper bounds $F, G, D$ and functions $M(\mathbf{x}, \delta)$ and $G_{L}$, are known, such that:

$|\Delta f|<F, \quad|\Delta g|<G, \quad|d|<D, \quad 0<G_{L}(\mathbf{x}) \leq g(\mathbf{x})$

$M(\mathbf{x}, \delta)=\left|x_{d}^{n}-\sum_{j=1}^{n-1} R_{j}^{n}(\delta) e^{(n-j)}\right|$

where $\Delta f=(f-\hat{f})$ and $\Delta g=(g-\hat{g})$.

Theorem 2 Consider the plant (1) and the sliding surface (23). If the values $F, G, D$ and $M$ as defined in assumption $\mathrm{I}$, then the sliding control law

$u=\hat{g}^{-1}\left[-\hat{f}+x_{d}^{n}-\sum_{j=1}^{n-1} R_{j}^{n}(\delta) e^{(n-j)}-\operatorname{Tsgn}(s)\right]$

where,

$T \geq \frac{\hat{g}(\mathbf{x})}{G_{L}(\mathbf{x})}\left[F+\frac{(M(\mathbf{x}, \delta)+|\hat{f}(\mathbf{x})|) G}{\hat{g}(\mathbf{x})}+D+\eta\right]$

guarantees the sliding condition, $\dot{s} \operatorname{sgn}(s) \leq-\eta$.

Proof. Consider the sliding surface

$s=\sum_{j=0}^{n-1} R_{j}^{n}(\delta) e^{(n-j-1)}=e^{(n-1)}+\sum_{j=1}^{n-1} R_{j}^{(n)}(\delta) e^{(n-j-1)}$

thus we have:

$\dot{s}=e^{(n)}+\sum_{j=1}^{n-1} R_{j}^{n}(\delta) e^{(n-j)}$ $\dot{s}=f+g\left[\hat{g}^{-1}\left(-\hat{f}+x_{d}^{(n)}-\sum_{j=1}^{n-1} R_{j}^{n}(\delta) e^{(n-j)}\right)\right]-$

$g \hat{g}^{-1} \operatorname{Tsgn}(s)-x_{d}^{(n)}+\sum_{j=1}^{n-1} R_{j}^{n}(\delta) e^{(n-j)}+d$

Therefore we have:

$\dot{s}=\Delta f-\left(g \hat{g}^{-1}-1\right) \hat{f}+\left(g \hat{g}^{-1}-1\right)\left(x_{d}^{(n)}-\sum_{j=1}^{n-1} R_{j}^{n}(\delta) e^{(n-j)}\right)$

$-g \hat{g}^{-1} T \operatorname{sgn}(s)+d$

(26) and (30) yield

$\dot{s} \operatorname{sgn}(s)=\left[\Delta f-\left(g \hat{g}^{-1}-1\right) \hat{f}+\left(g \hat{g}^{-1}-1\right)\left(x_{d}^{(n)}-\right.\right.$

$\left.\left.\sum_{j=1}^{n-1} R_{j}^{n}(\delta) e^{(n-j)}\right)+d\right] \operatorname{sgn}(s)-g \hat{g}^{-1} T \leq\left[\Delta f-\left(g \hat{g}^{-1}-1\right) \hat{f}\right.$

$\left.+\left(g \hat{g}^{-1}-1\right)\left(x_{d}^{(n)}-\sum_{j=1}^{n-1} R_{j}^{n}(\delta) e^{(n-j)}\right)+d\right] \operatorname{sgn}(s)$

$-\frac{g}{G_{L}}\left(F+\frac{(M+|\hat{f}|) G}{\hat{g}}+D+\eta\right)$

On the other hand, from assumption I, we have:

$\frac{g}{G_{L}}>1, \quad\left|g \hat{g}^{-1}-1\right| \leq G \hat{g}^{-1}$

By using the inequality of (31) and the relations of (32), we have:

$\dot{s} \operatorname{sgn}(s) \leq|\Delta f|+G \hat{g}^{-1}\left(\left|\left(x_{d}^{(n)}-\sum_{j=1}^{n-1} R_{j}^{n}(\delta) e^{(n-j)}\right)\right|+|\hat{f}|\right)+$

$|d|-\left(F+\frac{(M+|\hat{f}|) G}{\hat{g}}+D+\eta\right) \leq-\eta$

and this completes the proof.

Lemma 2: If $\delta$ and $R_{j}^{n}(\delta)$ are rejection parameter and rejection regulator, respectively, then

$\frac{\partial^{k}}{\partial \delta^{k}} R_{j}^{n}(\delta)=(-1)^{k} \frac{(n-1) !}{(n-k-1) !} R_{j-k}^{n-k}(\delta)$

Proof. This lemma is proved by induction method. Let $k=1$, thus:

$$
\begin{aligned}
& \frac{\partial}{\partial \delta} R_{j}^{n}(\delta)=\frac{\partial}{\partial \delta}\left(\begin{array}{c}
n-1 \\
j
\end{array}\right)(v-\delta)^{j}=-j\left(\begin{array}{c}
n-1 \\
j
\end{array}\right)(v-\delta)^{j-1} \\
& =-j\left(\frac{(n-1) !}{j !(n-j-1) !}\right)(v-\delta)^{j-1}=-\frac{(n-1)(n-2) !}{(j-1) !(n-2-j+1) !}(v-\delta)^{j-1} \\
& =-(n-1)\left(\begin{array}{c}
n-2 \\
j-1
\end{array}\right)(v-\delta)^{j-1}=-(n-1) R_{j-1}^{n-1}(\delta)
\end{aligned}
$$

Or 
Therefore Eq. (34) is satisfied for $k=1$. Assuming this relation is established for $k=p$, i.e:

$\frac{\partial^{p}}{\partial \delta^{p}} R_{j}^{n}(\delta)=(-1)^{p} \frac{(n-1) !}{(n-p-1) !} R_{j-p}^{n-p}(\delta)$

Now, let $k=p+1$. By using Eq (36) we have

$$
\begin{aligned}
& \frac{\partial^{p+1}}{\partial \delta^{p+1}} R_{j}^{n}(\delta)=\frac{\partial}{\partial \delta}\left(\frac{\partial^{p}}{\partial \delta^{p}} R_{j}^{n}(\delta)\right)=\frac{\partial}{\partial \delta}\left(\frac{(-1)^{p}(n-1) !}{(n-p-1) !} R_{j-p}^{n-p}(\delta)\right) \\
& =(-1)^{p} \frac{(n-1) !}{(n-p-1) !} \frac{\partial}{\partial \delta}\left(\begin{array}{c}
n-p-1 \\
j-p
\end{array}\right)(v-\delta)^{j-p}= \\
& (-1)^{p+1} \frac{(n-1) !}{(n-p-1) !}(j-p) \frac{(n-p-1) !(v-\delta)^{j-p-1}}{(j-p) !(n-p-1-j+p) !}= \\
& (-1)^{p+1} \frac{(n-1)(n-p-1)}{(n-p-1) !} \frac{(n-p-2) !(v-\delta)^{j-p-1}}{(j-p-1)(n-p-2-(j-p-1)) !}
\end{aligned}
$$

Therefore

$$
\frac{\partial^{p+1}}{\partial \delta^{p+1}} R_{j}^{n}(\delta)=(-1)^{p+1} \frac{(n-1) !}{(n-p-2) !} R_{j-(p+1)}^{n-(p+1)}(\delta)(37)
$$

and this completes the proof. $\square$

If we consider the sliding equation in the following form

$$
s=\sum_{j=0}^{n-1} R_{j}^{n}(\delta(t)) e^{(n-j-1)}
$$

where $\delta(t)$ is the time-variant rejection parameter, then this equation defines a timevariant sliding equation. In the next sections the sliding equation is assumed to be timevariant as defined in (38). Since in practical systems there are always uncertainties in the dynamical equations, the free models and identification methods are used in sliding mode control approach for improving the performance and avoiding the effects of perturbation and uncertainties in mathematical models. Wavelet networks, for approximation of nonlinear functions are well established [5], [6].

\section{Analysis}

\subsection{Wavelet networks}

Wavelets are especial kinds of functions which exhibit oscillatory behavior for a short period of time and then die out. They are obtained by using shift-invariance and dyadic dilation of a mother wavelet function $\psi \in V$ and extended by the basic properties of
Multi-Resolution Analysis [5]. Let $\psi$ be a mother wavelet function, that has a unit length. All the functions $\psi_{j, k}$ are defined as

$\psi_{j, k}(x)=2^{j / 2} \psi\left(2^{j} x-k\right), \quad j, k \in Z$

where $\psi_{j, k}$ have unit length also. If the family $\left\{\psi_{j, k}\right\}$ is an orthogonal basis of $V$ then we can define a wavelet series expansion that is uniformly convergent to $f(x) \in V$, in $L^{2}(R)$ [6]. That is

$\left\|f(x)-\sum_{j=-\infty}^{\infty} \sum_{k=-\infty}^{\infty} d_{j k} \psi_{j, k}(x)\right\|_{2}=0$

where

$d_{j k}=<f, \psi_{j, k}>=\int_{-\infty}^{\infty} f(x) \psi_{j, k}(x) d x$

wavelet network is a class of neural network consisting of wavelets. We can use the wavelet network for approximation of nonlinear functions. Let

$$
f(x, \boldsymbol{\theta})=\sum_{j=J_{1}=K_{1}}^{J_{2}} \sum_{j, k}^{K_{2}} \theta_{j, k} \psi_{j}(x)=\boldsymbol{\theta}^{T} \mathbf{W}(x), J_{i}, K_{i} \in Z
$$

Where

$\boldsymbol{\theta}=\left(\theta_{J_{1}, K_{1}}, \ldots, \theta_{J_{1}, K_{2}}, \ldots, \theta_{J_{2}, K_{1}}, \ldots, \theta_{J_{2}, K_{2}}\right)^{T} \in R^{N}$ as $\quad N=\left(J_{2}-J_{1}+1\right)\left(K_{2}-K_{1}+1\right)$ and

$\mathbf{W}(x)=\left(\psi_{J_{1}, K_{1}}, \ldots, \psi_{J_{1}, K_{2}}, \ldots, \psi_{J_{2}, K_{1}}, \ldots, \psi_{J_{2}, K_{2}}\right){ }^{T}$ The wavelet network $f(x, \boldsymbol{\theta})$ can be used to approximate $f(x)$ to any desired accuracy [6], [18]. By using the tuning laws and learning algorithms, the best values of $\theta_{j, k}$ for $j, k \in Z$ may be estimated such that desired accuracy is obtained. There are a number of methods for constructing multidimensional wavelets, in both single scaling and multiscaling forms. In the single scaling multidimensional wavelet, a single dilation parameter in all dimensions is used. In the multiscaling multidimensional wavelet, an independent dilation parameter in each dimension is used. A multidimensional wavelet can be built by tensor product of one dimensional wavelet function. i.e.:

$\psi(\mathbf{x})=\psi\left(x_{1}\right) \psi\left(x_{2}\right) . . \psi \nu\left(x_{m}\right)$ for $\mathbf{x}=\left(x_{1}, \ldots, x_{n}\right)$.

We have selected Mexican Hat wavelet function, as wavelet network basis functions. Accordingly, based on data set, the dilation 
value is chosen. Some wavelet candidates corresponding to various translation parameters are often redundant for constructing wavelet network approximator. So the OLS algorithm for purifying the wavelet candidate and estimating initial values of the wavelet network coefficients is used [18]. Translation parameters are considered as learning parameters of the network. In this paper, the EKF (Extended Kalman Filter) algorithm for incremental training the learning parameters is used. The tuning laws for adjusting the coefficients of wavelet expansion are presented in the next theorem. For designing the neural wavelet control, $u_{n w}$, wavelet approximations of the functions $f$ and $g$ are implemented. Also for adjusting the adaptive filters, rejection regulator are tuned by using the next theorem. Such away that the effects of the all un-modeled frequencies of the systems are reduced considerably, while preserving the stability and robustness of the systems. Consider the following sliding surface

$s=e^{n-1}+\sum_{j=1}^{n-1} R_{j}^{n}(\delta) e^{(n-j-1)}$.

According to the lemma 2 , we can write

$\sum_{j=1}^{n-1} \frac{\partial}{\partial \delta} R_{j}^{n}(\delta) e^{(n-j-1)}=-(n-1) \sum_{j=1}^{n-1} R_{j-1}^{n-1}(\delta) e^{(n-j-1)}$

therefore:

$\dot{s}=e^{n}+\sum_{j=1}^{n-1} R_{j}^{n}(\delta) e^{(n-j)}-(n-1) \dot{\delta} \sum_{j=1}^{n-1} R_{j-1}^{n-1}(\delta) e^{(n-j-1)}$

Substituting (1) into (46)

$\dot{s}=f(x)+g(x) u+d-x_{d}^{(n)}+\sum_{j=1}^{n-1} R_{j}^{n}(\delta) e^{(n-j)}-$

$-(n-1) \dot{\delta} \sum_{j=1}^{n-1} R_{j-1}^{n-1}(\delta) e^{(n-j-1)}$

Now we define

$u=\frac{1}{g_{n w}}\left[-f_{n w}+x_{d}^{n}-\sum_{j=1}^{n-1} R_{j}^{n}(\delta) e^{(n-j)}-T \Phi(\sigma, s)\right]$

In this paper, hyperbolic tangent function is used instead of saturation function, i.e.:

$\Phi(\sigma, s)=\tanh (\sigma s)$

such that $u_{n w}, f_{n w}=\boldsymbol{\theta}_{f}^{T} \cdot \mathbf{W}$ and $g_{n w}=\boldsymbol{\theta}_{g}^{T} \cdot \mathbf{W}$ are wavelet approximations of the ideal controller, $f$ and $g$ respectively. Also define the parameters $\boldsymbol{\theta}_{f}^{*} \in R^{N}$ and $\boldsymbol{\theta}_{g}^{*} \in R^{N}$ of the best function approximation as

$$
\begin{aligned}
& \boldsymbol{\theta}_{f}^{*}=\arg \min _{\boldsymbol{\theta}_{f} \in \Omega_{f}}\left\{\sup _{\mathbf{x} \in \Omega_{x}}\left\|f(\mathbf{x})-f_{n w}\left(\mathbf{x}, \boldsymbol{\theta}_{f}\right)\right\|\right\} \\
& \boldsymbol{\theta}_{g}^{*}=\arg \min _{\boldsymbol{\theta}_{g} \in \Omega_{g}}\left\{\sup _{\mathbf{x} \in \Omega_{x}}\left\|g(\mathbf{x})-g_{n w}\left(\mathbf{x}, \boldsymbol{\theta}_{g}\right)\right\|\right\}
\end{aligned}
$$

where

$\Omega_{f}=\left\{\boldsymbol{\theta}_{f}:\left\|\boldsymbol{\theta}_{f}\right\| \leq M_{f}\right\}$ and $\Omega_{g}=\left\{\boldsymbol{\theta}_{g}:\left\|\boldsymbol{\theta}_{g}\right\| \leq M_{g}\right\}$ are constraint sets of suitable bounds on $\boldsymbol{\theta}_{f}$ and $\boldsymbol{\theta}_{g}$, respectively, such that $M_{f}$ and $M_{g}$ are specified by designer. If $\boldsymbol{\theta}_{f}$ and $\boldsymbol{\theta}_{g}$ are estimates of $\boldsymbol{\theta}_{f}^{*}$ and $\boldsymbol{\theta}_{g}^{*}$, respectively, that are obtained by wavelet network approximator then we define

$$
f_{n w}^{*}(\mathbf{x})=\left(\boldsymbol{\theta}_{f}^{*}\right)^{T} \cdot \mathbf{W}(\mathbf{x}) \text { and } g_{n w}^{*}(\mathbf{x})=\left(\boldsymbol{\theta}_{g}^{*}\right)^{T} \cdot \mathbf{W}(\mathbf{x})
$$

Thus (47) can be rewritten as

$$
\begin{aligned}
& \dot{s}=\left(f_{n w}^{*}-f_{n w}\right)+\left(g_{n w}^{*}-g_{n w}\right) u+\xi_{f}+\xi_{g} u+d \\
& -(n-1) \dot{\delta} \sum_{j=1}^{n-1} R_{j-1}^{n-1}(\delta) e^{(n-j-1)}-s \operatorname{Ttanh}(\sigma s)
\end{aligned}
$$

where, $\quad u=u_{n w}, \quad \xi_{f}=f-f_{n w}^{*} \quad$ and $\xi_{g}=g-g_{n w}^{*}$. If we define $\boldsymbol{\theta}_{\widetilde{f}}=\boldsymbol{\theta}_{f}^{*}-\boldsymbol{\theta}_{f}$ and $\boldsymbol{\theta}_{\tilde{g}}=\boldsymbol{\theta}_{g}^{*}-\boldsymbol{\theta}_{g}$ then $\dot{\boldsymbol{\theta}}_{f}=-\dot{\boldsymbol{\theta}}_{\tilde{f}}, \dot{\boldsymbol{\theta}}_{g}=-\dot{\boldsymbol{\theta}}_{\tilde{g}}$ and also

$$
\dot{s}=\boldsymbol{\theta}_{\widetilde{f}}^{T} \mathbf{W}+\boldsymbol{\theta}_{\widetilde{g}}^{T} \mathbf{W} u+\varepsilon(t)-(n-1) \dot{\delta} \sum_{j=1}^{n-1} R_{j-1}^{n-1}(\delta) e^{(n-j-1)}
$$

$-s \operatorname{Ttanh}(\sigma s)$

so that, $\varepsilon(t)=\xi_{f}+\xi_{g} u+d$.

Theorem 3: Consider a nonlinear system and the sliding surface described by Eqs (1) and (44), respectivelly. If the wavelet robust sliding-mode controller is designed as equation (48) and (49), in which the following tuning laws

$$
\dot{\boldsymbol{\theta}}_{f}=\left\{\begin{array}{cc}
\eta_{1} s \mathbf{W} & i f\left(\left\|\boldsymbol{\theta}_{f}\right\|<M_{f}\right) \\
& \operatorname{or}\left(\left\|\boldsymbol{\theta}_{f}\right\|=M_{f},\left(s \mathbf{W}^{T} \boldsymbol{\theta}_{f} \leq 0\right)\right) \\
P_{f} & i f\left(\left\|\boldsymbol{\theta}_{f}\right\|=M_{f},\left(s \mathbf{W}^{T} \boldsymbol{\theta}_{f}>0\right)\right)
\end{array}\right.
$$




$$
\dot{\boldsymbol{\theta}}_{g}=\left\{\begin{array}{cc}
\eta_{2} s \mathbf{W} u & i f\left(\left\|\boldsymbol{\theta}_{g}\right\| k M_{g}\right) \\
& \operatorname{or}\left(\left\|\boldsymbol{\theta}_{g}\right\| M_{g},\left(s u \mathbf{W}^{T} \boldsymbol{\theta}_{g} \leq 0\right)\right) \\
P_{g} & i f\left(\left\|\boldsymbol{\theta}_{g}\right\| M_{g},\left(s u \mathbf{W}^{T} \boldsymbol{\theta}_{g}>0\right)\right)
\end{array}\right.
$$

where $P_{f}$ and $P_{g}$ are projection operators, that is defined as

$$
P_{f}=\eta_{1} s \mathbf{W}-\frac{\eta_{1} s \mathbf{W}^{T} \boldsymbol{\theta}_{f}}{\left\|\boldsymbol{\theta}_{f}\right\|^{2}} \boldsymbol{\theta}_{f} \quad \eta_{1}>0 .
$$

and

$$
P_{g}=\eta_{2} s \mathbf{W} u-\frac{\eta_{2} s u \mathbf{W}^{T} \boldsymbol{\theta}_{g}}{\left\|\boldsymbol{\theta}_{g}\right\|^{2}} \boldsymbol{\theta}_{g} \quad \eta_{2}>0 .
$$

and also

$$
\dot{\delta}=\eta_{3} \operatorname{sgn}(s) \sum_{j=1}^{n-1} R_{j-1}^{n-1}(\delta) e^{(n-j-1)} \quad \eta_{3}>0 .
$$

and

$$
\dot{T}=\eta_{4} \operatorname{stanh}(\sigma . s), \quad \quad \eta_{4}>0 .
$$

are defined, then tracking error will converge to zero asymptotically.

Proof: Consider the Lyapunov function in the following form

$$
V=\frac{1}{2} s^{2}+\frac{1}{2 \eta_{1}} \boldsymbol{\theta}_{\tilde{f}}^{T} \boldsymbol{\theta}_{\widetilde{f}}+\frac{1}{2 \eta_{2}} \boldsymbol{\theta}_{\tilde{g}}^{T} \boldsymbol{\theta}_{\tilde{g}}+\frac{1}{2 \eta_{3}} T^{2}
$$

Differentiating equation (61), with respect to time, yields

$$
\begin{aligned}
& \dot{V}=s \dot{s}+\frac{1}{\eta_{1}} \boldsymbol{\theta}_{\tilde{f}}^{T} \dot{\boldsymbol{\theta}}_{\tilde{f}}+\frac{1}{\eta_{2}} \boldsymbol{\theta}_{\tilde{g}}^{T} \dot{\boldsymbol{\theta}}_{\tilde{g}}+\frac{1}{\eta_{3}} T \dot{T} \\
& \dot{V}=s\left(\boldsymbol{\theta}_{\tilde{f}}^{T} \cdot \mathbf{W}+\boldsymbol{\theta}_{\tilde{g}}^{T} \cdot \mathbf{W} u+\varepsilon(t)-(n-1) \dot{\delta} \sum_{j=1}^{n-1} R_{j-1}^{n-1}(\delta) e^{(n-j-1)}\right)- \\
& -s \operatorname{Ttanh}(\sigma s)+\frac{1}{\eta_{1}} \boldsymbol{\theta}_{\tilde{f}}^{T} \dot{\boldsymbol{\theta}}_{\tilde{f}}+\frac{1}{\eta_{2}} \boldsymbol{\theta}_{\bar{g}}^{T} \dot{\boldsymbol{\theta}}_{\tilde{g}}+\frac{1}{\eta_{3}} T \dot{T}= \\
& =\boldsymbol{\theta}_{\tilde{f}}^{T}\left(s \mathbf{W}+\frac{1}{\eta_{1}} \dot{\boldsymbol{\theta}}_{\tilde{f}}\right)+\mathbf{\theta}_{\tilde{g}}^{T}\left(s \mathbf{W} u+\frac{1}{\eta_{2}} \dot{\boldsymbol{\theta}}_{\tilde{g}}\right)+s(\varepsilon(t))- \\
& -s(n-1) \dot{\delta} \sum_{j=1}^{n-1} R_{j-1}^{n-1}(\delta) e^{(n-j-1)}+\left(\frac{1}{\eta_{3}} T \dot{T}-\operatorname{sTtanh}(\sigma s)\right)
\end{aligned}
$$

Since $\dot{\boldsymbol{\theta}}_{f}=-\dot{\boldsymbol{\theta}}_{\tilde{f}}$ and $\dot{\boldsymbol{\theta}}_{g}=-\dot{\boldsymbol{\theta}}_{\tilde{g}}$ therefore by implementing the equations (55)-(60) in equation (63), we have

$$
\dot{V} \leq-|s|(n-1)\left[\sum_{j=1}^{n-1} R_{j-1}^{n-1}(\delta) e^{(n-j-1)}\right]^{2}
$$

$+|s||\varepsilon(t)|$

If $\mathbf{e}(0)$ is bounded then according to the theorem 1, the sliding function (44) with Hurwitz characteristic polynomial is bounded. So $\mathbf{e}(t)$ and $\sum_{j=1}^{n} R_{j-1}^{n-1}(\delta) e^{(n-j-1)}$ are bounded. Therefore $s \in L_{\infty}$ and there exists a real positive number $M_{\delta}$, such that

$$
\left|R_{j-1}^{n-1}(\delta) e^{(n-j-1)}\right| \leq \frac{M_{\delta}}{\sqrt{n-1}} .
$$

Relation (64) can be rewritten as:

$\dot{V} \leq-|s| \cdot M_{\delta}^{2}+|s||\varepsilon(t)|$

By integrating both sides of (66) and by some manipulation, we have

$$
\int_{0}^{t}|s(\tau)| d \tau \leq \frac{1}{M_{\delta}^{2}}[V(0)+V(t)]+\frac{|s|}{M_{\delta}^{2}} \int_{0}^{t}|\varepsilon(\tau)| d \tau .
$$

According to the universal approximation theorem and the assumption of bounded external disturbances, $x$ is bounded and $\varepsilon \in L_{1}$. If $\varepsilon \in L_{1}$ then from (67) we have $s \in L_{1}$. Moreover, the adaptive laws in (55) and (56) guarantee that $\boldsymbol{\theta}_{f} \in \Omega_{f}$ and $\boldsymbol{\theta}_{g} \in \Omega_{g}$. This implies that all variables on the right hand side of (46) are bounded, therefore $\dot{s} \in L_{\infty}$. By using the corollary of Barbalat's Lemma [14], we have $|s| \rightarrow 0$, as $t \rightarrow \infty$. From (66), if $\varepsilon=0$, it is clear that $\dot{V} \leq-|s| \cdot M_{\delta}^{2}<0$ else, the fact that $|s| \rightarrow 0$ implies that $\dot{V} \leq 0$. From (66) and (44) only possible condition for $\dot{V}=0$ when $|s| \rightarrow 0$, is $e=0$. In this case, the tracking error is or approach to zero and this completes the proof.

\subsection{Fuzzy boundary layer}

In the sliding-mode control, the abrupt change of sign of the term $T s g n(s)$ produces chattering. To avoid this drawback, the boundary layer from both sides of the sliding surface $s=0$ is introduced. If the state of system is out of the boundary layer, then by using the reaching condition the state reaches to the inside of the boundary layer. The fixed boundary layer usually does not guarantee the precise tracking. So, in this paper, a varying boundary layer is considered. It is clear that, absolute value of the control signal is 
dependent to distance $d$ between the actual state and the line perpendicular to the switching surface $s=0$. Such that, $|u|$ should increase as $d$ grows [10]. According to (44), let

$$
\mathbf{a}_{N}=\frac{\left(R_{0}^{n}(\delta), R_{1}^{n}(\delta), \ldots, R_{n-1}^{n}(\delta)\right)^{T}}{\left\|\left(R_{0}^{n}(\delta), R_{1}^{n}(\delta), \ldots, R_{n-1}^{n}(\delta)\right)\right\|}
$$

be the unit normal vector of the sliding surface in the $(n-1)$-dimentional phase plane. Therefore we have:

$s=\left\|\left(R_{0}^{n}(\delta), R_{1}^{n}(\delta), \ldots, R_{n-1}^{n}(\delta)\right)\right\| \mathbf{a}_{N} \cdot \mathbf{e}$

According to the Eq. (69), $s$ is a multiple of the projection of the state vector $\mathbf{e}$ on the direction of the vector $\mathbf{a}_{N}$, which is the directional distance from $\mathbf{e}$ to the sliding surface. Therefor the shortest Euclidean distance $d$ between $\mathbf{e}$ and the direction of $\mathbf{a}_{N}$ is:

$$
d(\delta, \mathbf{e})=\sqrt{\|\mathbf{e}\|^{2}-s^{2}}
$$

Thus, the distance $d$ is dependent to rejection parameter, $\delta$ and the error state vector. By using the tuning law in (59), $\delta$ is tuned subject to the tracking error be decreased. Therefore $s$ and $d$ are adjusted so that $d$ be decreased. Now by using the following fuzzy system the proper variable boundary layer parameter $\sigma$ is estimated.

$$
\text { IF } s_{f u z z y} \text { is Lingvar THENG } \sigma_{f u z z y} \text { is Lingvar(71) }
$$

Where $s_{f u z z y}$ and $\sigma_{f u z z y}$ are fuzzified values of $s$ and $\sigma$, respectively, that have any linguistic variables (Ling.var), as is shown in the following rule base table:

Table 1. Fuzzy rule base.

\begin{tabular}{|l|l|l|l|l|}
\hline$s_{\text {fuzzy }}$ & ZE & SM & ME & BI \\
\hline$\sigma_{\text {fuzzy }}$ & BI & ME & SM & ZE \\
\hline
\end{tabular}

Where the sets of fuzzy quantities are ZE: zero, SM: small, ME: medium and BI: big. This fuzzy system adopts absolute value of sliding surface $|s|$, as input and the boundary layer parameter $\sigma$, as output. This fuzzy system tunes the boundary layer width in the control law. Figure 2 shows the effect of variation of $\sigma$ on the boundary layer width. As $\sigma$ gets larger the width of boundary layer becomes narrower. Figure 3 shows the configuration of the wavelet robust control by fuzzy boundary layer via time-variant sliding surface.

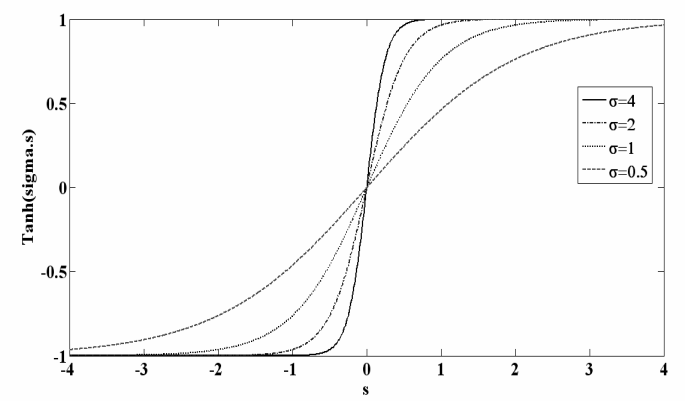

Figure 2. Variation of the boundary layer. parameter

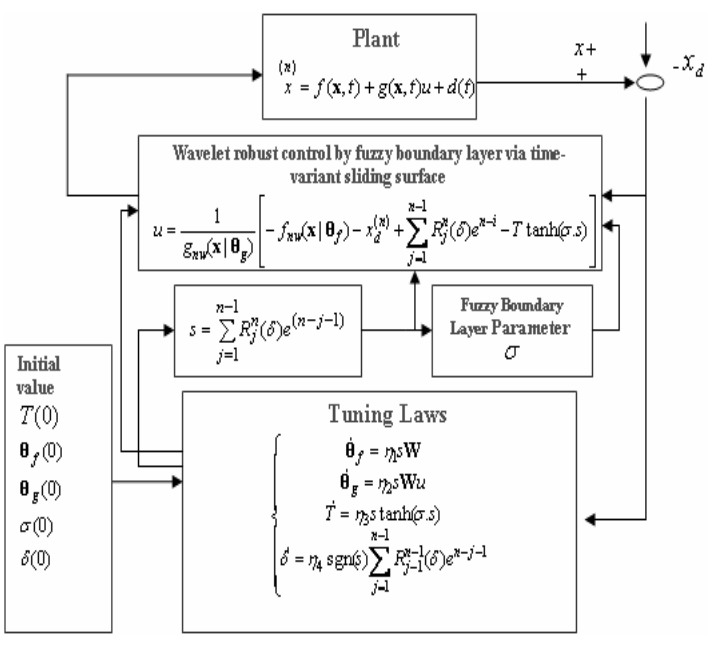

Figure 3. Configuration of the wavelet robust control by fuzzy boundary layer via time-variant sliding surface.

\section{Example}

The inverted pendulum is a classic problem in dynamics and control theory. It is widely used as benchmark for testing control algorithms. In this section an example in inverted pendulum plant is presented. We compare WRCFBL method and the methods presented in [4] and [8]. Without loss of generality, we consider $v=20$.

\subsection{Formulation}

The dynamic equation of the inverted pendulum system, is stated in the following: 


$$
\begin{aligned}
\ddot{x} & =\frac{g \sin (x)-\frac{m l \dot{x}^{2} \cos (x) \sin (x)}{M+m}}{L\left(\frac{4}{3}-\frac{m \cos (x)^{2}}{M+m}\right)}+ \\
& +\left(\frac{\frac{\cos (x)}{M+m}}{l\left(\frac{4}{3}-\frac{m \cos (x)^{2}}{M+m}\right)}\right) u+d
\end{aligned}
$$

where $g=9.8 m / s^{2}, \quad M$ and $m$ are the acceleration of gravity, the mass of the cart and the mass of the pole, respectively, and $L$ is half-length of the pole. To demonstrate the performance of the presented method, the Mexican Hat function $\psi(x)=\left(1-x^{2}\right) e^{-x^{2} / 2}$ as our wavelet basis function is selected. Accordingly, based on data set $x_{d}$, dilation and translation parameters are assigned as $j \in\{-5,-4, \ldots, 4,5\} \quad$ and $\quad k \in\{-10, \ldots, 10\}$, respectively. After implementing the OLS and EKF algorithms for purifying and training the wavelet network parameters, the WRCFBL tuning laws for tuning the network coefficients, are implemented. The performance index is the integral of absolute error:

$$
I A E=\int_{0}^{\infty}|e(t)| d t .
$$

Table 2. Dilation value and number of translations.

\begin{tabular}{|l|l|l|l|l|l|}
\hline Dilation parameter, $\mathrm{j}$ & 0 & 1 & 2 & 3 & 4 \\
\hline Number of translation & 1 & 2 & 4 & 8 & 10 \\
\hline
\end{tabular}

For designing the fuzzy boundary layer rule base, the membership function for $|\mathbf{s}|$ and $\delta$ are given in Figures 4 and 5, respectivelly. The simulation results are shown in Figures 6-12. Figures 6 and 7 show that the state of system tracks the desired trajectory quicker than in [4] and [8] while, the absolute value of the control signal is less than in [4] and [8] (see Table 3). The proposed controller is robust to the time-variant mass of the cart and length of the pole. Figure 8 shows the variation of rejection parameter $\delta$, for tuning the chain of the $(n-1)$ adaptive first-order low- pass filters in (44). Figure 9 shows the variation of sliding function. Figure 10 indicates the variation of the fuzzy boundary layer parameter, $\sigma$. Figures 11 and 12 indicate the variation of the control gain parameter and the performance index, IAE. To illustrate the effectiveness of the proposed method, the results of this example are compered with those of other methodologies in Table 3.

Table 3 Benchmarks for controller performance.

\begin{tabular}{|c|c|c|c|}
\hline Criterion & GA_MAFSMC[4] & AFSMC [8] & The proposed method \\
\hline Number of fuzzy rules & - & 36 & 4 \\
\hline Control signal, $u$ & $-13.2 \leq u \leq 396$ & $-120 \leq u \leq 15.701$ & $-109.4425 \leq u \leq 12.4255$ \\
\hline IAE & 0.4753 & 0.37201 & 0.3414 \\
\hline
\end{tabular}

Let the desired trajectory be $x_{d}=0$. For the convenience of simulation, the external disturbance $d$ is assumed to be a square wave with amplitude \pm 0.05 and period $2 \pi$. Let, $L=0.5+0.3 \sin (30 t), M=1+0.05 \sin (30 t)$

\subsection{Results}

The initial parameter values are selected as $x(0)=7 \pi / 18, \quad \dot{x}(0)=0, \quad \delta(0)=3, \quad \eta=10$, $\sigma(0)=3$, and $T(0)=36.4$. Also, let $M_{f}=30$ and $M_{g}=20$. The dilation value, $\mathrm{j}$, and the number of translation parameters for each dilation, presented by OLS method, are given

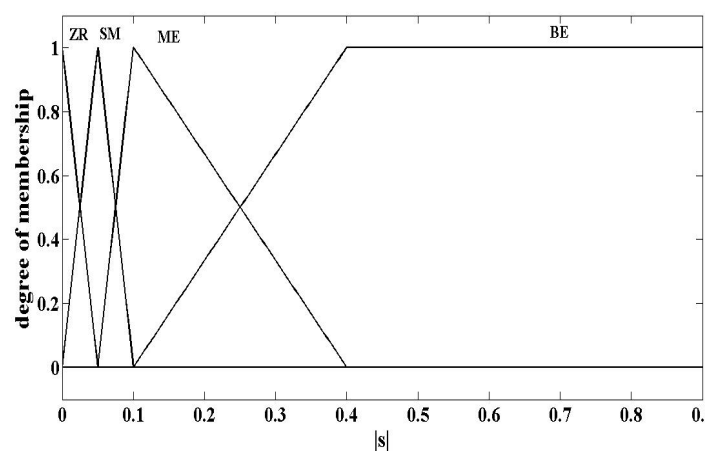

Figure 4. The membership function for $|s|$. 


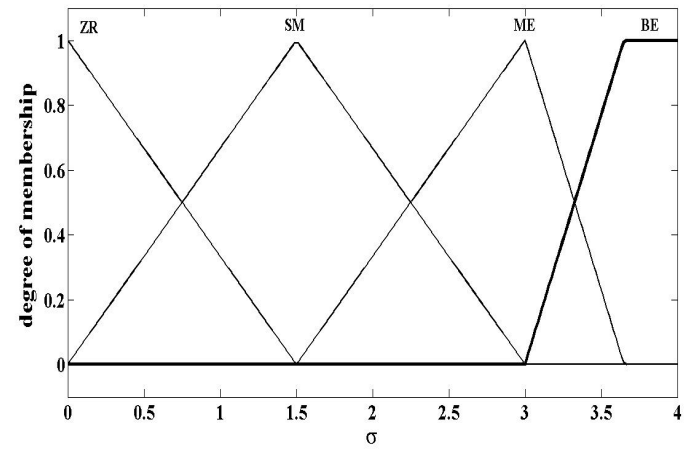

Figure 5. The memberships function for boundary layer parameter.

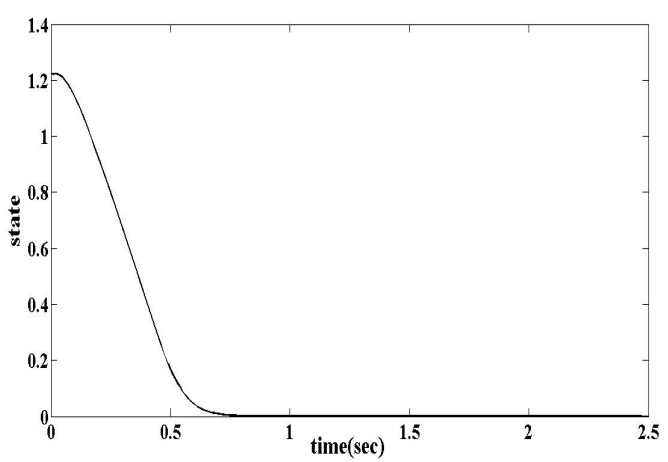

Figure 6. Tracking state.

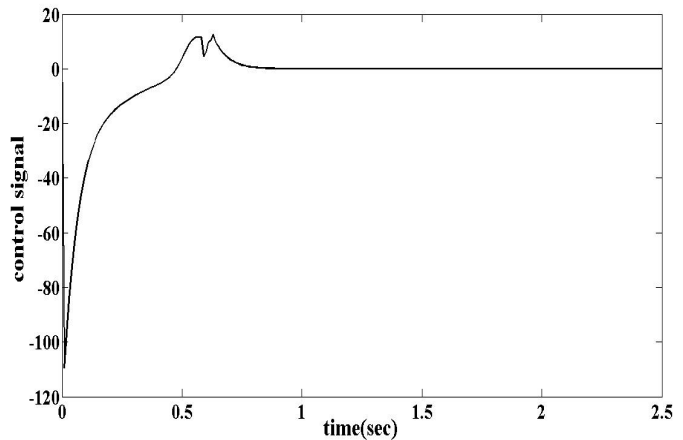

Figure 7. Control signal.

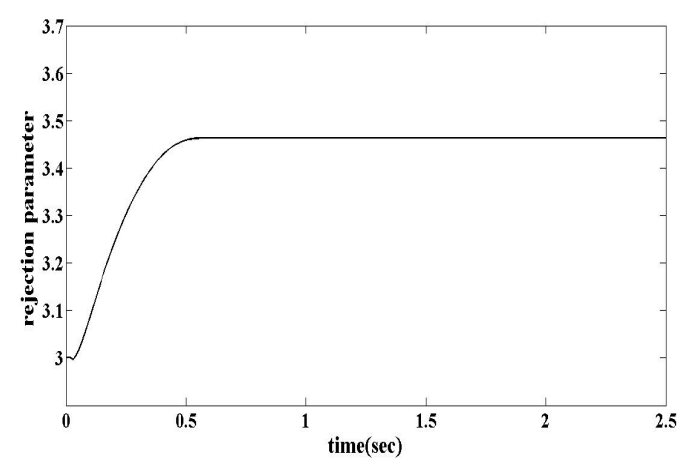

Figure 8. Rejection parameter.

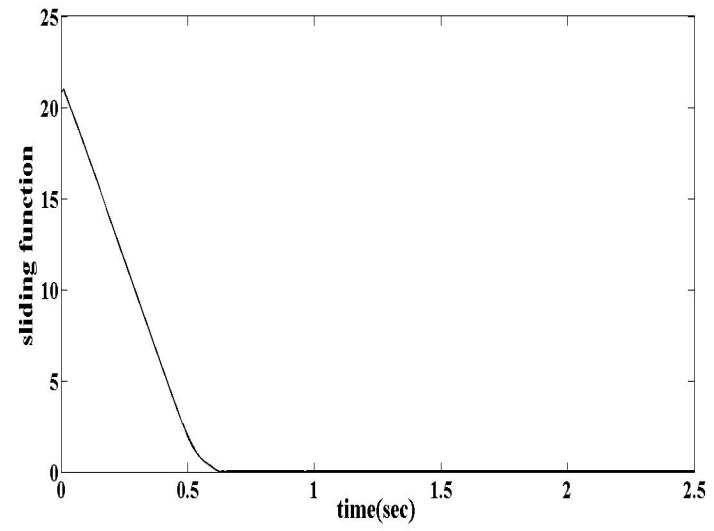

Figure 9. Sliding surface.

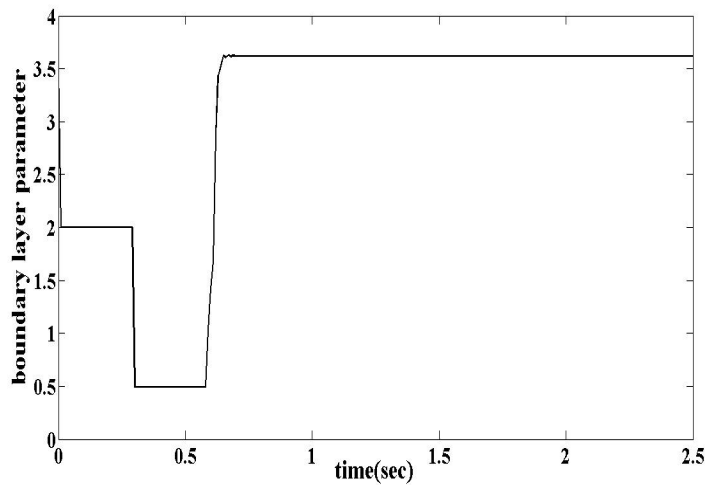

Figure 10. Boundary layer parameter.

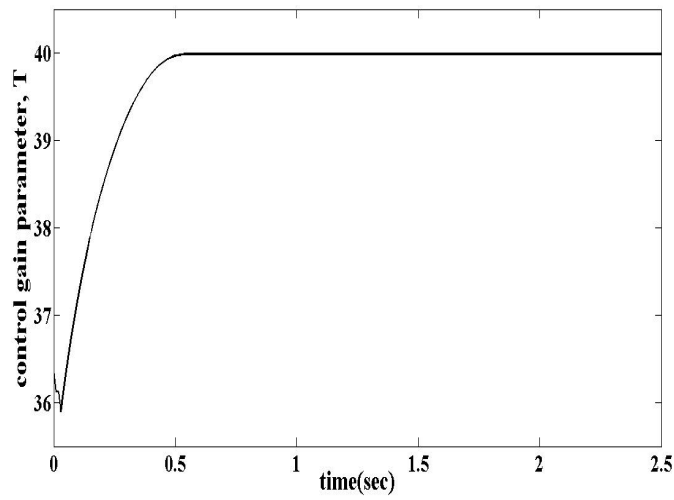

Figure 11. Control gain parameter.

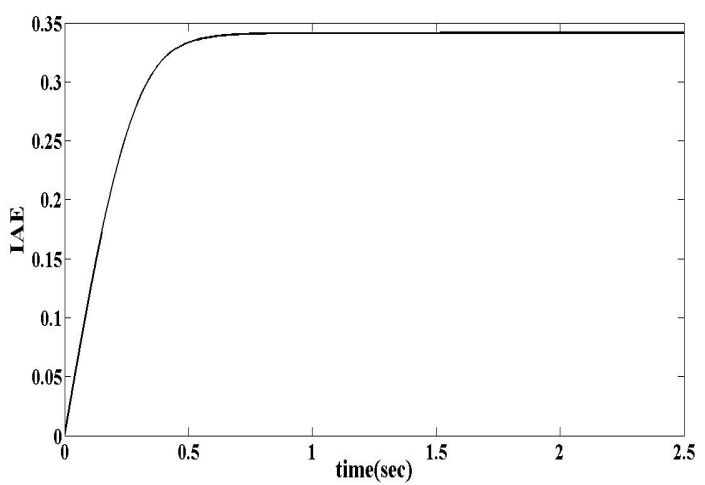

Figure 12. The integral of absolute error. 


\section{Conclusion}

In this paper, a wavelet robust control by fuzzy boundary layer via time-variant sliding surface for a class of uncertain nonlinear systems is proposed. New terminologies, rejection parameter and rejection regulator, those are adjustable, for defining the timevariant sliding surface are introduced. Wavelet network based on the multiscale analysis, synthesis properties and the learning abilities presents the well approximation of nonlinear functions. The wavelet network coefficients, control gain parameter and rejection regulator are tuned independent of a priori knowledge of the system. Since, the tracking precision is not guaranteed by using the saturation function, so, instead of the saturation function a hyperbolic tangent function is used. A fuzzy system that adopts absolute value of sliding surface as input and the boundary layer parameter as output is defined. Control system stability is guaranteed using the Lyapunov method. By using the proposed controller, based on the time-variant sliding equation and varying boundary layer, all un-modeled frequencies are adaptively filtered. Also the effects of the wavelet approximation errors and the external disturbance on tracking performances are attenuated efficiently. The control input chattering does not occur. Simulation example illustrates the superior performance and the advantages of the proposed method.

\section{REFERENCES}

1. ABDEL-HADY, F., S. M. ABUELENIN, Design and Simulation of a Fuzzysupervised PID Controller for a Magnetic Levitation System, Studies in Informatics and Control, September vol. 17, 2007, pp. 315-328.

2. BABUSKA, R., H. VERBRUGGEN, Neuro-fuzzy Method for Non-linear System Identification, Annual Review in Control, vol. 27, 2003, pp. 73-85.

3. CHANG, W. D., R. C. HWANG, J. G. HSIEH, Application of an Auto-tuning Neuron to Sliding-mode Control, IEEE Transaction on System, Man and Cybernetics, vol. 32, 2002, pp. 517-529.
4. CHEN, P. C., C. W. CHEN, W. L. CHIANG, GA-based Modified Adaptive Fuzzy Sliding Mode Controller for Nonlinear system, Expert Systems With Applications, vol. 36, 2009, pp. 5872-5879.

5. CHUI, C. K, An Introduction to Wavelets, Academic Press, 1992.

6. DELYON, B, A. JUDITSKY, A. BENVENISTE, Accuracy Analysis for Wavelet Approximations, IEEE Transaction on Neural Networks, vol. 6, no. 2, 1995, pp. 332-348.

7. FINKBEINER, D. T, Introduction to Matrices and Linear Transformation, Third Edition, Dehli, Shahdra, 1986.

8. Ho, H. F., Y. K. WONG, A. B. RAD, Adaptive Fuzzy Sliding Mode Control with Chattering Elimination for Nonlinear SISI Systems, Simulation Modeling Practice and Theory, vol. 17, 2009, pp. 1199-1210.

9. KUO, T. C., S. H. SHANG, Slidingmode Control with Self-tuning Law for Uncertain Non-linear Systems, ISA, Transactions, vol. 47, 2008, pp. 171-178.

10. PALM, R, Robust Control by Fuzzy Sliding Mode, Automatica, vol. 9, 1994, pp. 1429-1437.

11. HSU, C. F., T. T. LEE, C. M. LIN, L. Y. CHEN, Robust Neuro-fuzzy Controller Design via Sliding-mode Approach, IEEE Proceeding of Budapest, Hungary Conf., 2004, pp. 917-922.

12. LEE, H., E. KIM, H. J. KANG, A New Sliding-mode Control with Fuzzy Boundary Layer, Fuzzy Sets and Systems, vol. 120, 2001, pp. 135-143.

13. PHAN, P. A., T. J. GALE, Direct Adaptive Fuzzy Control with a Selfstructuring Algorithm, Fuzzy Sets and Systems, vol. 159, 2008, pp. 871-899.

14. Slotine, J. J., W. LI, Applied Nonlinear Control. Englewood Cliffs, New Jersey, 1991.

15. SRIVASTAVA, S., M. SINGH, M. HANMMANDLU, A. N. JHA, New Fuzzy Wavelet Neural Networks for System Identification and Control, 
Applied Soft Computing, vol. 6, 2005, pp. 1-17.

16. TURNER, M. C., D. G. BATES, Mathematical Methods for Robust and Non-linear Control, Springer Berlin Heidelberg New York, 2007.
17. WANG, C. H., T. C LIN, T. T. LEE, H. L. LIU, Adaptive Hybrid Intelligent Control for Uncertaine Non-linear Dynamical Systems, IEEE Transaction on System, Man, and Cybernetics, vol. 32 , no. 5, 2002, pp. 583-597.

18. ZHANG, Q, Using Wavelet Network in Nonparametric Estimation, IEEE Trans. Neural Networks, vol. 8, 1992, pp. 227-236. 